Society of Automotive Engineers (SAE) Technical Paper 2006-01-1041

Paper presented at SAE 2006 World Congress \& Exposition, April 5, 2006, Detroit, MI

Published in Combustion and Flow Diagnostics and Fundamental Advances in Thermal Fluid Sciences 2006. Vol. SP-2015. (Society of Automotive Engineers, Troy, MI, 2006, ISBN 0-7680-1750-5) 


\title{
Quantitative Characterization of Near-Field Fuel Sprays by Multi-Orifice Direct Injection Using Ultrafast $X$-Tomography Technique
}

\author{
Xin Liu, Kyoung-Su Im, Yujie Wang, Jin Wang \\ Argonne National Laboratory, Argonne, IL 60439 \\ David L.S. Hung, James R. Winkelman \\ Visteon Corporation, Van Buren Township, MI 48111
}

\author{
Mark W. Tate ${ }^{1}$, Alper Ercan ${ }^{2}$, Lucas J. Koerner ${ }^{1}$, Thomas Caswell ${ }^{1}$, Darol Chamberlain ${ }^{3}$, \\ Daniel R. Schuette ${ }^{1}$, Hugh Philipp ${ }^{1}$, Detlef M. Smilgies ${ }^{3}$, Sol M. Gruner ${ }^{1,3}$ \\ ${ }^{1}$ Department of Physics, ${ }^{2}$ Department of Applied and Engineering Physics, ${ }^{3}$ Cornell High Energy Synchrotron Source, \\ Cornell University, Ithaca, NY 14853
}

Copyright @ 2006 SAE International

\section{ABSTRACT}

A low-pressure direct injection fuel system for spark ignition direct injection engines has been developed, in which a high-turbulence nozzle technology was employed to achieve fine fuel droplet size at a low injection pressure around $2 \mathrm{MPa}$. It is particularly important to study spray characteristics in the nearnozzle region due to the immediate liquid breakup at the nozzle exit. By using an ultrafast $x$-ray area detector and intense synchrotron $x$-ray beams, the interior structure and dynamics of the direct injection gasoline sprays from a multi-orifice turbulence-assisted nozzle were elucidated for the first time in a highly quantitative manner with $\mu$ s-temporal resolution. Revealed by a newly developed, ultrafast computed x-microtomography technique, many detailed features associated with the transient liquid flows are readily observable in the reconstructed spray. Furthermore, an accurate 3dimensional fuel density distribution, in the form of fuel volume fraction, was obtained by the time-resolved computed tomography. The time-dependent fuel density distribution revealed that the fuel jet is well broken up immediately at the nozzle exits. These results not only reveal the near-field characteristics of the partial atomized fuel sprays with unprecedented detail, but also facilitate the development of an advanced multi-orifice direct injector. This ultrafast tomography capability also will facilitate the realistic computational fluid dynamic simulations in highly transient and multiphase fuel spray systems.

\section{INTRODUCTION}

Technologies capable of improving fuel efficiency and reducing emissions play an essential role in the design of the new-generation automotive internal combustion engines as the worldwide demand for energy grows rapidly [1]. Among them, gasoline direct injection (GDI) has been the subject of research and development for a long time in the automotive industry. In a combustion system employing GDI, the fuel is injected directly into the combustion chamber instead of the air-intake port. Due to the ability to precisely control the injection rate, timing, and combustion of the fuel, the fuel efficiency and emission reduction potentials can be greatly improved [2].

The design of the fuel injector plays a key role in the performance of GDI engines. Compared to the conventional port fuel injection (PFI) engines, the available space and time for fuel evaporation and mixing in GDI engines are inevitably reduced. This requires the injector to deliver sprays with sufficiently small droplet size and large flow rates for high-load conditions. Consequently, high-pressure direct-injection (HPDI) fuel systems and swirl injectors dominate GDI engine applications worldwide because they can achieve both requirements simultaneously. However, this technology also has many inherent drawbacks, such as high system cost, complicated system configuration, excessive wall wetting, and lack of spray tailoring flexibility [3]. To overcome these potential shortcomings and to maximize the benefits afforded by the GDI concept, a low-pressure direct-injection (LPDI) fuel injector was developed, which 
utilizes a high-turbulence nozzle to produce lowpressure "soft" sprays, with droplet size comparable to the HPDI swirl injector sprays, but with much reduced system complexity and cost [2-4].

Turbulence nozzles have been investigated since the 1980s [5,6] as a viable means to promote atomization and produce small fuel droplets in addition to HPDI swirl injectors. The distinctive feature of the current type of injector lies in the turbulence generated inside the nozzle, which significantly disturbs the initial liquid jet formed at the nozzle even at low injection pressure, e.g., at $2 \mathrm{MPa}$. The turbulence induces prompt breakup at the nozzle exit and the whole atomization process therefore is accelerated. To ensure an adequate flow rate at such a low injection pressure, multi-orifice nozzle plates are normally used. Figure 1 schematically shows the configuration of a nozzle plate with 9 conical orifices, which is currently used in the LPDI system.

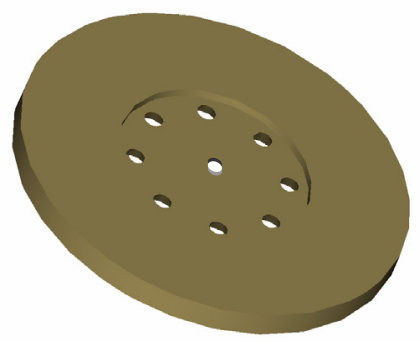

Figure 1. Schematic of a 9-orifice nozzle plate used in the LPDI system. The diameter of the outer side of each conical orifice is about $350 \mu \mathrm{m}$.

In the past few years, extensive experiments have been carried out to quantify the characteristics of "soft" sprays generated by similar multi-orifice LPDI systems, such as injector flow rate, far-field spray droplet size, and spray angle and penetration [3,4]. Despite significant advances in laser diagnostic techniques in the past decades, the optical measurements in the near-nozzle and densespray region (i.e., near-field) still have not yielded the desired quantitative information primarily due to the optical multiple scattering from dense fuel droplets [7-9]. In this particular case, the prompt break up at the nozzle exit, the near-field sprays already contain large amounts of droplets and cannot be simply assumed as intact liquid. Therefore, quantitative characterization of sprays in this region is particularly interesting. Recently, a new nonintrusive, quantitative, and time-resolved technique to characterize the dense part of fuel sprays has been developed using a synchrotron-radiation-based monochromatic x-radiography/tomography technique [10-14]. This technique takes advantage of the high intensity and monochromaticity of the x-rays, allowing accurate quantitative measurements of highly transient fuel sprays in a time-resolved manner.

In this paper, we demonstrate the application of ultrafast x-tomography on near-field multi-orifice LPDI sprays. A robust fast-Fourier algorithm has been used to reconstruct the spray structure and dynamics in three dimensions (3D) and as a function of injection time.

\section{EXPERIMENTS, ANALYSIS, AND RESULTS}

\section{MULTI-ORIFICE FUEL INJECTOR}

It has been shown in a previous study [4] that multiorifice fuel injectors offer more flexibility in tailoring the spray patterning compared to other existing fuel injectors using either swirl or slit type nozzles. This flexibility is achieved by independently controlling orifice quantity, patterns, and orientation on the multi-orifice nozzle plate, all of which can be precisely designed to target individual spray plumes and to control the overall spray distribution among all the plumes. In addition, the geometry of the internal flow cavity can also be designed in a flexible way. Utilizing advanced manufacturing technologies, the nozzle's geometrical parameters and internal structure can be fabricated with high precision and minimal part-to-part variation.

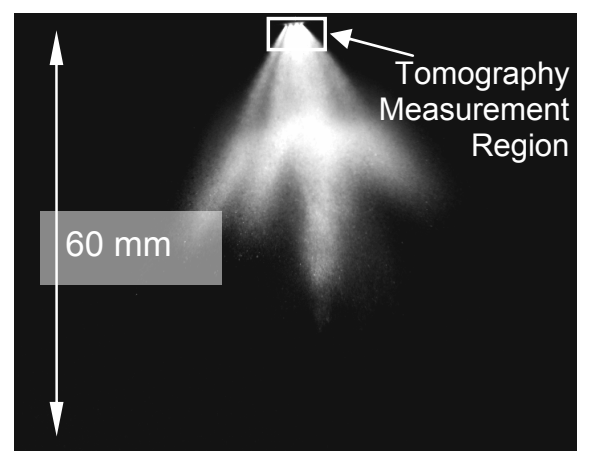

Figure 2. Mie-Scattering spray image of the 9-orifice fuel injector tilted towards the right by $5^{\circ}$.

Figure 2 shows a Mie-scattering image of this 9-orifice fuel injector spray recorded at $2.5 \mathrm{~ms}$ after the start of injection (SOI). Direct illumination on the spray was provided by a strobe light located at a slight angle from the direction of the camera. Due to packaging constraints, fuel injectors are commonly installed onto the cylinder head in a position where the spray pattern and axis may not be at an optimal orientation with respect to the cylinder and/or piston geometry. This is particularly true for a cylinder head with a side-mounted injector location. In this situation, the fuel-spray axis may need to be inclined in order to avoid fuel impingement on valves or cylinder walls and to provide better fuel-air mixing for different operation modes of the engine [4]. Therefore, as part of the spray tailoring flexibility, it is necessary to implement a spray offset angle so that the spray axis can be inclined with respect to the injector body axis. Such an offset spray pattern is depicted in Fig. 2 and is produced by the injector at a normal $60^{\circ}$ full spray angle and a $5^{\circ}$ axial-offset angle tilted counterclockwise from the injector axis for this specific 


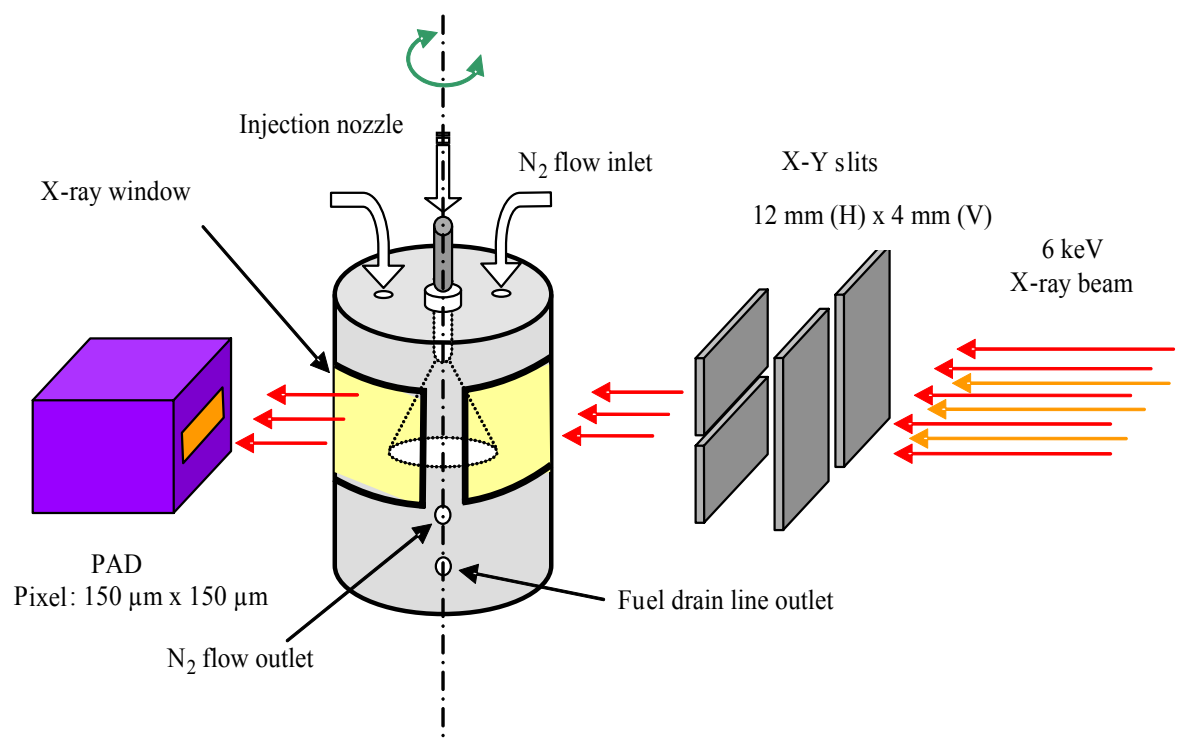

Figure 3. Schematic of experimental setup.

imaging geometry. The small region highlighted in Fig. 2 illustrates the imaging area where the ultrafast $x-$ tomography measurements were performed.

\section{EXPERIMENTS}

The experiments were performed at the $\mathrm{D}-1$ beamline of the Cornell High Energy Synchrotron Source (CHESS). Figure 3 shows a schematic of the experimental setup. The $\mathrm{x}$-ray beam produced by synchrotron radiation was monochromatized to $6.0 \mathrm{keV}$ (with an energy bandwidth of about $1 \%$ ) using a double-multilayer monochromator. This x-ray energy is optimal for probing the fuel, a blend of a calibration fluid and a fuel additive containing cerium. The calibration fluid (Viscor ${ }^{\circledR} 16-A$ ) is a simulated fuel with properties similar to gasoline fuel with precisely controlled viscosity and specific gravity specifications. The monochromized beam was further collimated by a set of slits of size $12 \mathrm{~mm}$ (Horizontal) $\times 4$ $\mathrm{mm}$ (Vertical).

The key component in the setup is the integrated tomography fuel chamber system, which includes the spray injection chamber and rotation and translation stages. The injection chamber is intended to provide an environmental enclosure for the fuel sprays. As shown in Fig. 3, there are two identical X-ray transparent windows situated symmetrically on the chamber with a $120^{\circ} \mathrm{x}$-ray viewing angle. The windows are made of thin polymer films. The injector was mounted in a mounting plate on the top of the chamber. The injection pressure was set at $2 \mathrm{MPa}$, and the nominal pulse duration of the spray is $2.5 \mathrm{~ms}(1.5-\mathrm{ms}$ net pulse duration with a 1-ms precharging duration). Also fit to the chamber were two inlets and one outlet for flowing nitrogen gas through the chamber to scavenge the fuel vapor. On the side of the chamber was a fuel drain line. The environment in the spray chamber was maintained at a pressure of $0.1 \mathrm{MPa}$ and at room temperature $\left(25-30^{\circ} \mathrm{C}\right.$ in the radiation enclosure).

The spray chamber is designed to rotate and to translate in precise steps while the x-ray source and the detector are stationary. In this system, we use a horizontal rotational stage and a vertical translational stage to rotate and translate the spray chamber and to select the slice to be imaged in the vertical direction. The minimum rotation angle is $0.0025^{\circ}$, and the minimum step size for the translation stage is $1.27 \mu \mathrm{m}$. All the rotational and translational stages are motorized. During the experiment, the parallel $\mathrm{x}$-ray beams luminated the spray at a given view angle $\theta$, and after completion of the scans in temporal steps, the injection nozzle is rotated a small angle increment $\Delta \theta$ (normally $1^{\circ}$ ) and the temporal scan is repeated. This process is continued until a $180^{\circ}$ rotation is completed.

Another important component in the setup is the ultrafast $x$-ray detector, pixel array detector (PAD) developed at

Table 1. Experimental conditions

\begin{tabular}{ll}
\hline Parameters & Quantity and Properties \\
\hline Injection system & LPDI, multi-orifice nozzle \\
Orifice size & $0.35 \mathrm{~mm}$ outer diameter \\
Fill gas & $\mathrm{N}_{2}, 0.1 \mathrm{MPa}, 25 \sim 30^{\circ} \mathrm{C}$ \\
Fuel & Viscor $\AA$ with Ce-additive $4 \% \mathrm{wt}$ \\
Fuel density & $857.7 \mu \mathrm{g} / \mathrm{mm}^{3}$ \\
Pulse duration & $2.5 \mathrm{~ms}($ nominal) \\
Injection pressure & $2 \mathrm{MPa}$ \\
Region of interest & $0 \sim 8 \mathrm{~mm}$ from the nozzle \\
\hline \hline
\end{tabular}


Cornell University $[15,16]$. The pixel size of the PAD is $150 \mu \mathrm{m} \times 150 \mu \mathrm{m}$. We used a total of $92(\mathrm{H}) \times 40(\mathrm{~V})$ pixels on the sensor chip, limited by the $x$-ray beam size, as the individual imaging frame size. The complete imaging area was built up by shifting the position of the injector relative to the beam and the PAD. During the experiment, the spray was triggered at $1.15 \mathrm{~Hz}$ and a series of frames was taken at various delay times. The exposure time per frame was set to $10.25 \mu \mathrm{s}$ with an interval between frames of $25.6 \mu \mathrm{s}$. Each image was obtained by averaging 20 fuel-injection cycles. The complete experiment conditions are summarized in Table 1.
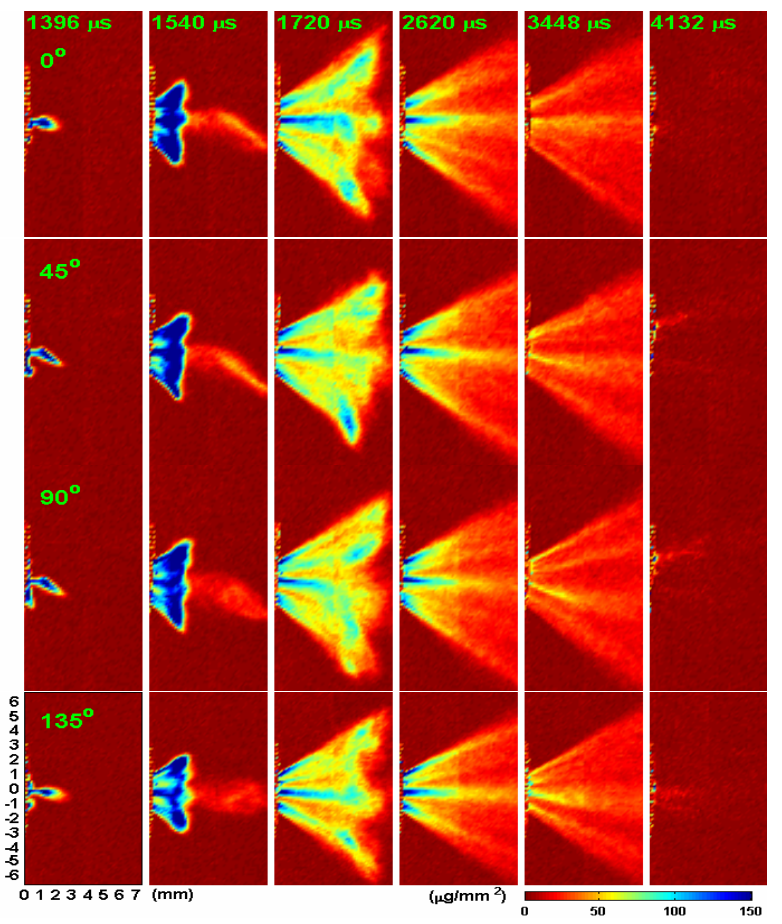

Figure 4. X-radiography snapshots of 9-orifice GDI sprays at several projection angles $\left(0^{\circ}, 45^{\circ}, 90^{\circ}\right.$, and $135^{\circ}$, selected from a total of 180 projections angles with $1^{\circ}$ interval) and different time instances: $1396 \mu \mathrm{s}$, when the center-orifice jet started exiting the nozzle; $1540 \mu \mathrm{s}$, when the "sac" exited from all the orifices; $1720 \mu \mathrm{s}$, when the "sac" fuel started to break up with the primary jets; $2620 \mu \mathrm{s}$, when the sprays were in a steady state; $3448 \mu \mathrm{s}$, just after the nozzles started to close; $4132 \mu \mathrm{s}$, when all the orifices were closed. All time instances were measured from SOI.

In Fig. 4, two-dimensionally projected radiography images of the 9-orifice GDI spray are shown at different time instances and different projection angles. Again, the nozzle plate is tilted by $5^{\circ}$ from injector body axis as shown in Fig. 2. Note that the imaging intensity has been converted to the $2 \mathrm{D}$ projected fuel mass density in units of $\mu \mathrm{g} / \mathrm{mm}^{2}$, after a proper density calibration described previously [10]. These high-contrast images demonstrate the details of dynamic characteristics of the mass distribution of the multi-orifice gasoline spray at near-nozzle regions, including time-dependent irregular density distribution and spray variations from one orifice to another, never before reported in a such quantitative manner by conventional optical or non-optical spray diagnostic techniques.

\section{NUMERICAL ANALYSIS}

From the projection images, the spray mass distribution in the form of fuel density or fuel volume fraction can be reconstructed in 3D by the computerized tomography (CT) technique [17]. The mathematical description of the fuel-spray CT is built upon the experimental geometry shown in Figs. 3 and 5. In a cross-sectional view perpendicular to the injector body, which coincides with the rotation axis, consider a parallel $\mathrm{x}$-ray beam transmitting an object (the hollow cone spray for illustration) in Fig. 5. The function $f(x, y)$ represents the position-dependent $x$-ray linear attenuation coefficients, where $(x, y)$ are the Cartesian coordinates fixed on one cross-sectional slice of the spray. A set of laboratory coordinates $(r, s)$ is also defined as shown in Fig. 5, while $(x, y)$ can rotate about their origin. With a rotational angle $\theta$, the two coordinate systems can be transformed by:

$$
\left[\begin{array}{l}
x \\
y
\end{array}\right]=\left[\begin{array}{ll}
\cos \theta & \sin \theta \\
-\sin \theta & \cos \theta
\end{array}\right]\left[\begin{array}{l}
r \\
s
\end{array}\right] .
$$

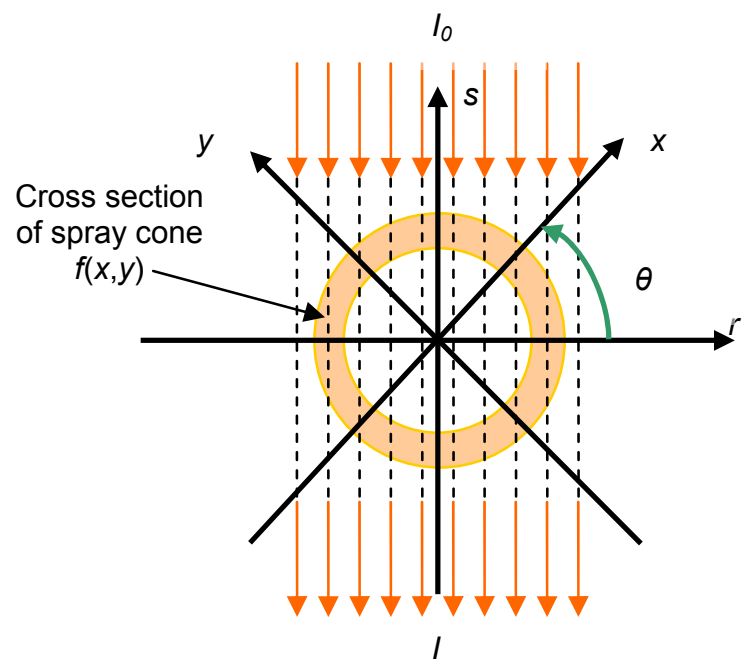

Figure 5. Definition of spray and laboratory coordinate systems.

Since monochromatic $x$-ray beams are used, the transmitted $x$-ray intensity profile $I$ as a function of $r$ at given angle $\theta$ is simply described by:

$$
\begin{aligned}
I(r, \theta) & =I_{0} \exp \left[-\int_{s} f(x, y) d s\right] \\
& =I_{0} \exp \left[-\int_{s} f(r \cos \theta+s \sin \theta,-r \sin \theta+s \cos \theta) d s\right],
\end{aligned}
$$


where $I_{0}$ is the incident $\mathbf{x}$-ray beam intensity and $d s$ is the spatial interval element along the beam path. The sinogram $p(r, \theta)$ in polar coordinates $(r, \theta)$ is defined by:

$$
\begin{aligned}
p(r, \theta) & =-\ln \frac{I(r, \theta)}{I_{0}} \\
& =\int_{s} f(r \cos \theta+s \sin \theta,-r \sin \theta+s \cos \theta) d s,
\end{aligned}
$$

which is the mathematical description of the imaging data set recorded by the PAD.

With the sinogram, we can reconstruct $f(x, y)$ by several numerical methods based on filtered back-projection (FBP), algebraic reconstruction, and the Fourier transform algorithms. The algebraic reconstruction method is the slowest algorithm of the three methods, but it can be used when the tomographic data are not collected with uniform angular intervals, which is not used in our reconstruction. The results given by the FBP method and the Fourier transform method are very close in our experiment, but the latter has proven to be the most efficient algorithm. Thus, we chose the Fourier transform algorithm as the preferred reconstruction method. With the central slice theorem (CST), one can reconstruct $f(x, y)$ through the Fourier transform and the inverse Fourier transform as shown in equation (4):

$$
f(x, y)=F^{-1}\{F[f(x, y)]\}=F^{-1}\{F[p(r, \theta)]\},
$$

where $F$ and $F^{-1}$ are the Fourier transform and the inverse Fourier transform, respectively. The fuel mass density distribution $\rho(x, y)$ is simply derived by:
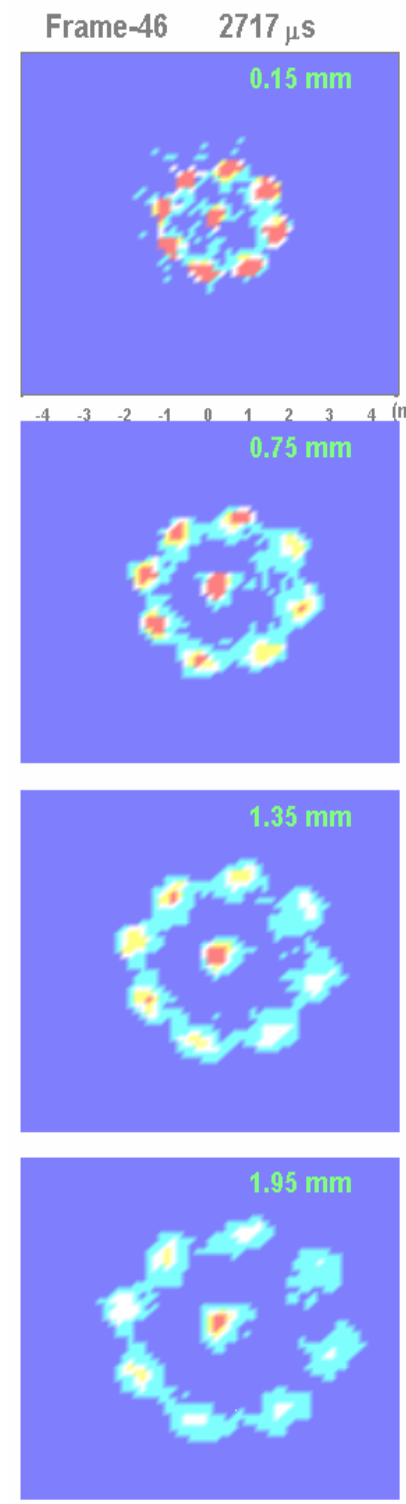
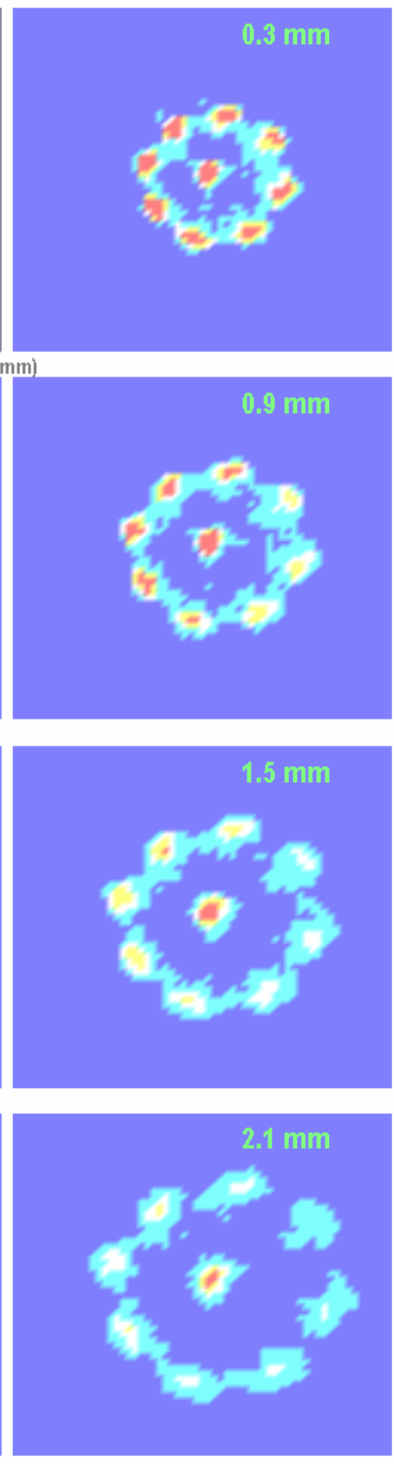
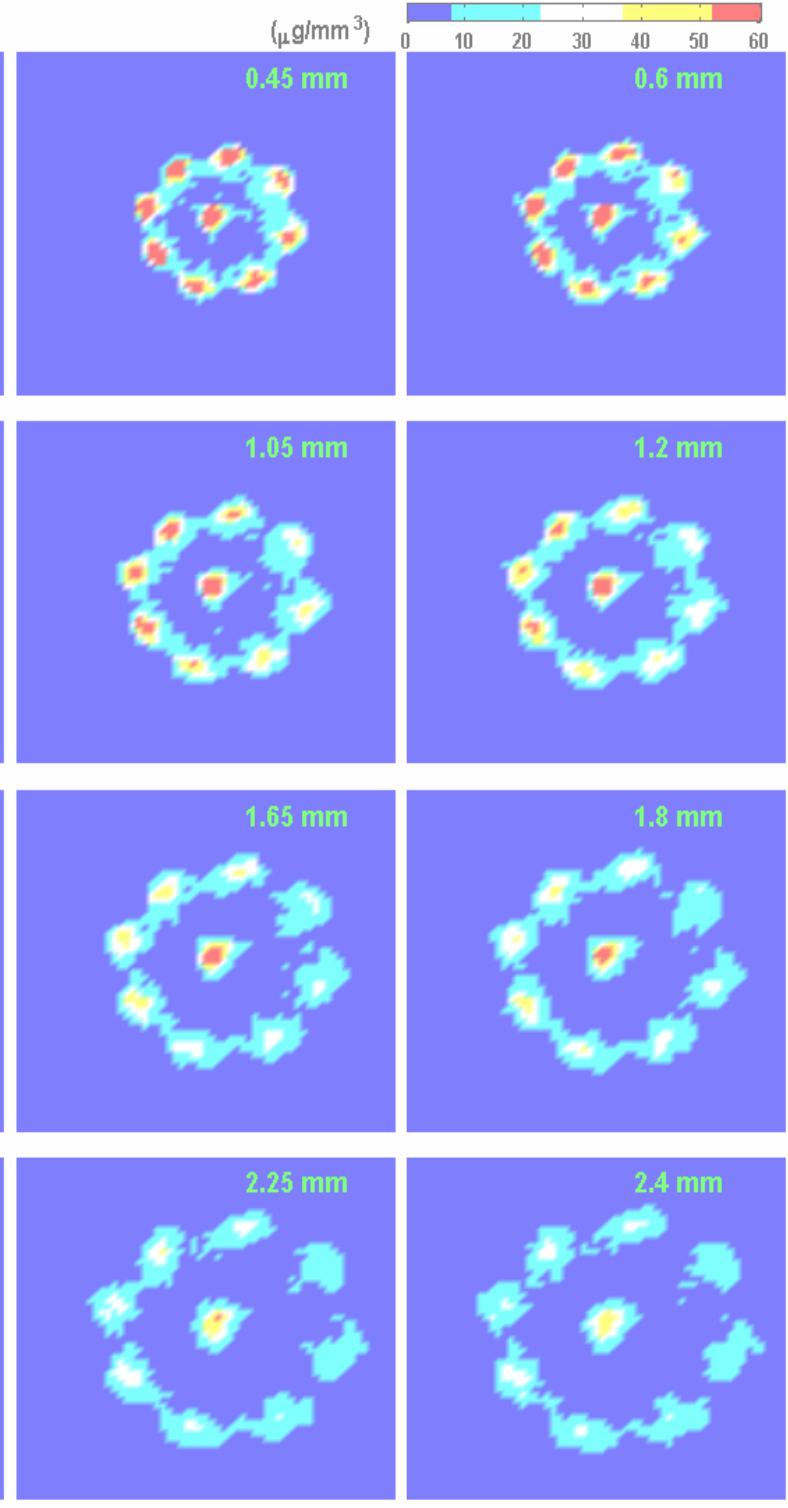

Figure 6. Reconstructed fuel density distribution at $2717 \mu$ s after SOI and at different locations from the nozzle. 


$$
\rho(x, y)=\frac{f(x, y)}{\mu_{M}},
$$

where $\mu_{M}$ is the mass attenuation coefficient of the fuel, which is evaluated through a calibration procedure.

The next experimental parameter that needs to be determined is the minimum number of view angles. This can be achieved by considering the reconstruction resolution and the limited data collection time. The number of viewing angles is also dependent on the size of the viewing area. Larger viewing areas may need more view angles. By equating the radial resolution and the worst-case azimuthal resolution, the minimum number of view angles required is estimated by [15]:

$$
N_{v}=\frac{\pi}{2} N_{p},
$$

where $N_{v}$ is the number of viewing angles and $N_{p}$ is the number of data pixels. For example, there are 92 pixels horizontally to record the projections in the PAD, resulting in a minimum $N_{v}$ of 145 . In the actual experiment, 180 view angles are used.

\section{RESULTS AND DISCUSSION}

Tomographic reconstruction is a great improvement over radiographic imaging and an absolute necessity for nonaxially symmetric sprays. The flow pattern of the multiorifice sprays has been revealed in a highly quantitative manner, which is very difficult to achieve by other means. Consequently, quantitative characterization of each jet from the different nozzle orifice can be performed. Figure 6 shows the reconstructed 9-orifice nozzle fuel density distribution of a single slice at different positions and $2.7 \mathrm{~ms}$ after SOI when the steady portion of the spray is being formed. This figure indicates that all 9 jets from the corresponding orifices can be clearly identified. Even though each single jet has somewhat irregular shapes, it appears to have a relatively dense core region surrounded by a cloud of possible liquid/gas mixture. This absorption-based tomography technique can not distinguish the liquid and gas phases, but it can give an accurate fuel density, which can be transformed to the volume fraction of the fuel (either in liquid or gas form). The diameter of each single jet increases while the jet peak density, which is only $40 \%$ of the bulk liquid density $\left(857.7 \mu \mathrm{g} / \mathrm{mm}^{3}\right)$ even at the orifice exit, falls off rapidly along each spray axis. Note the intensity of the images has a unit of $\mu \mathrm{g} / \mathrm{mm}^{3}$, a true 3D fuel mass density.

Figure 7 shows a more detailed spray cross-sectional reconstruction $1.8 \mathrm{~mm}$ from the nozzle exit. The fuel density distribution is plotted on a polar coordinate to illustrate the locations of individual jets in the overall spray structure. This mass distribution shows that the tilted nozzle plate caused the overall spray pattern to be slightly shifted from the injector's center axis as well as the tomography axis. This confirms the tilted feature of the offset spray depicted in Fig. 2. This asymmetry leads to the jets on the left side apparently having a higher density distribution than the right side (i.e., jets 3 , 4, and 5 have higher density than jets 1,7 , and 8 ).

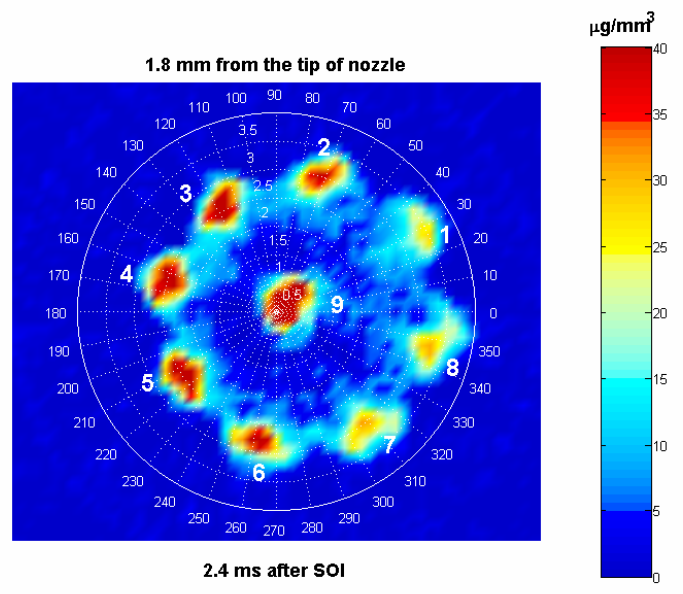

Figure 7. Reconstructed spray cross section plotted in polar coordinates. The cross section is positioned 1.8 $\mathrm{mm}$ downstream from the nozzle exit, $2.4 \mathrm{~ms}$ after the SOI during the steady part of the spray.

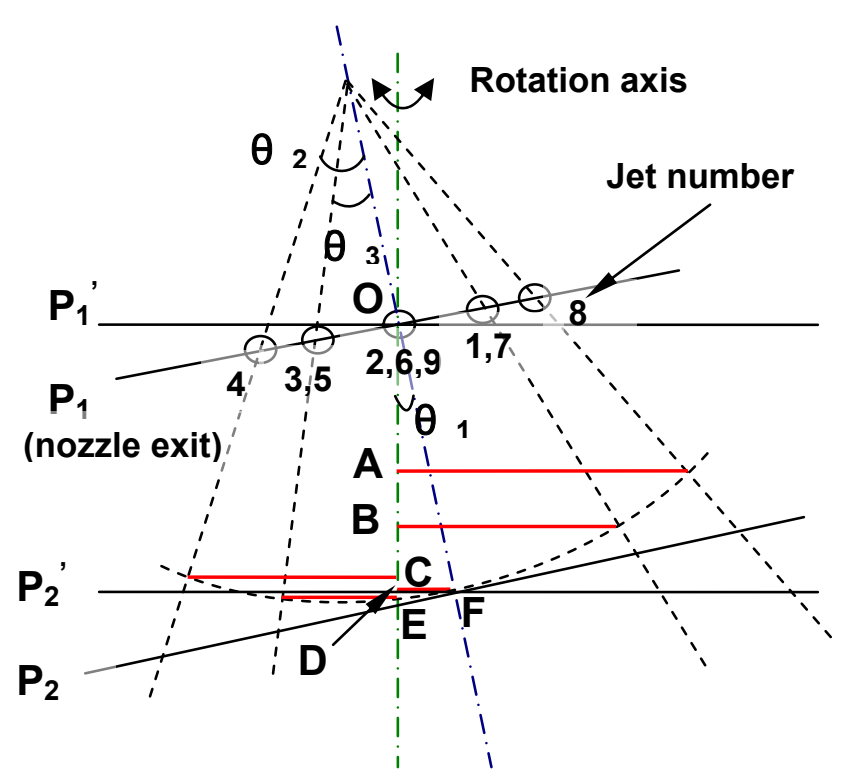

Figure 8. Diagram of the projected view of the tilted multi-orifice injection. At the viewing angle, orifice groups $(3,5),(2,6,9)$, and $(1,7)$ are overlapping.

The study of individual jet characteristics and orifice-toorifice spray variation is particularly important to the design and optimization of multi-orifice nozzles. With the quantitative $3 \mathrm{D}$ reconstruction, considerable information can be obtained. To compare each individual jet, the tilting effect should first be considered schematically, as a projected view specified in Fig. 8 . The nozzle plate $\left(P_{1}\right)$ 
is tilted counterclockwise with a tilt angle $\theta_{1} \cdot \theta_{2}$ is the half cone angle, which is $30^{\circ}$ in this case. Based on Fig. 7 , the nine jets are divided into five groups as shown in Fig. 8. $P_{2}$ represents a plane parallel to the nozzle plate $P_{1}$ at a certain downstream distance $\overline{O F}$. Planes $\mathrm{P}_{1}{ }^{\prime}$ and $\mathrm{P}_{2}{ }^{\prime}$ represent the cross sections of tomography reconstruction, which are perpendicular to the rotation axis. A reasonable comparison should be made at an equal linear distance traveled by each jet. Since the tomography reconstruction is $3 \mathrm{D}$, the density distribution of each jet at any position can be retrieved. To simplify the process, only the geometry center of each jet is considered here. As shown in Fig. 8, each point of the jet geometry center is back-projected to the vertical axis, such that $\overline{O A}, \overline{O B}, \overline{O C}, \overline{O D}$, and $\overline{O E}$ are the corresponding distances of the tomography cross section for each jet. Referring to the geometry shown in Fig. 8, the correlation between the projected distances and the linear distance $\overline{O F}$ are shown below:

$$
\begin{aligned}
& \overline{O A}=\overline{O F} \cdot \cos \left(\theta_{1}+\theta_{2}\right), \\
& \overline{O B}=\overline{O F} \cdot \cos \left(\theta_{1}+\theta_{3}\right), \\
& \overline{O C}=\overline{O F} \cdot \cos \left(\theta_{2}-\theta_{1}\right), \\
& \overline{O D}=\overline{O F} \cdot \cos \theta_{1}, \\
& \overline{O E}=\overline{O F} \cdot \cos \left(\theta_{3}-\theta_{1}\right),
\end{aligned}
$$

where, $\theta_{1}, \theta_{2}$, and $\theta_{3}$ are $5^{\circ}, 30^{\circ}$, and $22.2^{\circ}$, respectively. Considering the small tilt angle and limited distance we are studying, $\overline{O C}, \overline{O D}$, and $\overline{O E}$ are very close to $\overline{O F}$. In addition, the spatial resolution of the measurement is about $150 \mu \mathrm{m}$, such that the four distances are nearly indistinguishable when $\overline{O F}$ is less than $1.6 \mathrm{~mm}$. On the contrary, the differences between $\overline{O A}$ and $\overline{O F}$, as well as $\overline{O B}$ and $\overline{O F}$, are significant. This explains why the density distribution of jets 2, 3, 4, 5, 6, and 9 look similar and much different than jets 1, 7, and 8 in Fig. 7.

A time-evolution of selected peak fuel volume fraction of several jets is shown in Fig. 9, indicating that the spray started with a "sac" after the needle valve lift. Immediately after the "sac" there was a short timedependent fluctuation. The main body of the spray was relatively steady. One interesting observation is that the jet from the center orifice behaved quite unlike the eight jets from the periphery orifices. It had much higher fuel volume fraction than the other jets; the fluctuation after the "sac" was more transient; and it started and finished earlier than the surrounding jets, presumably due to the nozzle inner geometry. In addition, when the armature closed against the valve seat, there was a very small "bounce" for the center jet while no "bounce" was observed for the other eight peripheral jets. Also in Fig. 9, the peak fuel volume fraction of jet 8 , both with and without distance correction, are plotted. Although the location of the peak fuel volume fraction is not necessarily the same location as the geometric center of each jet, relationships illustrated in equation (7) are still a good approximation for the distance correction, considering the limited spatial resolution.

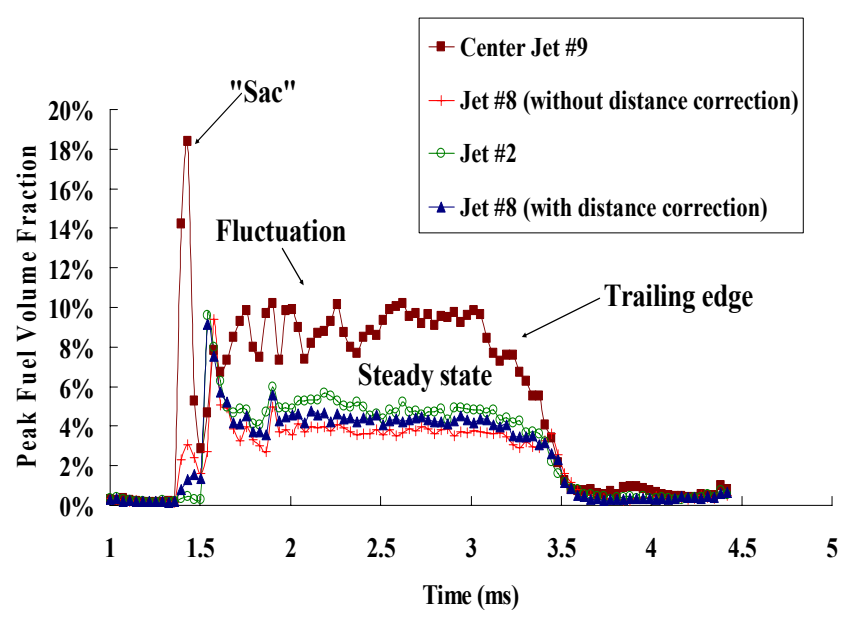

Figure 9. Time evolution of peak fuel volume fraction of selected jets at $1.8 \mathrm{~mm}$ from center axis of nozzle.

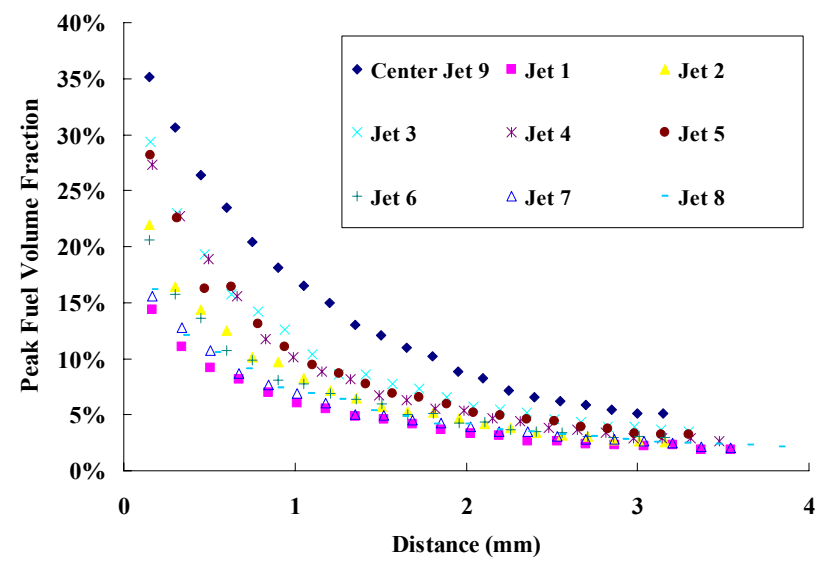

Figure 10. Falloff of peak fuel volume fraction of each jet along the spray axis. The data represent the fuel density at $2.6 \mathrm{~ms}$ after the SOI during the steady portion of the spray.

Figure 10 shows the peak fuel volume fraction of each jet at steady state as a function of downstream distance. The tilt effect is removed in this plot based on equation (7). Again, this figure shows the center jet has the highest peak fuel volume fraction distribution along the downstream distance among all the jets. It is noted that the peak fuel volume fraction at the nozzle exit is about $40 \%$, which verified the prompt jet breakup at the nozzle exit. Note that the peak fuel volume fraction distributions of the eight peripheral jets, even with distance correction, can be different. This is caused by the internal nozzle geometry as designed. 


\section{CONCLUSION}

We demonstrated the use of monochromatic $x$ tomography to study the near-field multi-orifice GDI sprays in a highly quantitative and time-resolved manner. The time evolution of the sprays was directly imaged with microsecond temporal resolution, and the internal structure of the multi-orifice spray was fully reconstructed quantitatively with submillimeter spatial resolution. The preliminary results indicate that the core region near the nozzle is composed of a liquid/gas mixture with a density much less than the bulk liquid fuel density. The breakup immediately at the nozzle exit due to the turbulence is verified, and the fuel volume fraction is about $40 \%$. The distribution of the pure fuel volume fraction along the spray axis falls off within $3 \mathrm{~mm}$ from $40 \%$ to $10 \%$. This technique allows the quantitative determination of several key transient spray characteristics such as the "sac," the fluctuation, and orifice-to-orifice spray variation. Additional key information about single-jet characteristics, orifice-toorifice jet atomization, and coalescence can also be derived with further analysis. The information obtained in this experiment should benefit the theoretical simulation of multi-orifice spray formation processes in this nearfield region. The success of the measurements has demonstrated that the $x$-tomography technique is well suited for multi-orifice spray characterization in the close proximity of the injector tip. We also believe that this technique can be used as a sensitive probe and diagnostic tool for investigating other highly transient phenomena.

\section{ACKNOWLEDGMENTS}

The authors are thankful to Robert Larson for his initiative effort. Technical assistance from Seong-Kyun Cheong and Christopher F. Powell is greatly appreciated. The work is supported by the U.S. Department of Energy, Office of Science, Office of Basic Energy Sciences, under contract W-31-109-ENG-38 and by an Argonne National Laboratory internal grant. The authors wish to acknowledge the technical support from Visteon Corp. The authors also thank the staff at CHESS, which is funded by the U.S. National Science Foundation and the U.S. National Institute of General Medical Sciences via NSF under award DMR-9713424. PAD detector development was funded by DOE grants DE-FG-0297ER62443.

\section{REFERENCES}

1. Cohn, D.R., and Heywood, J.B., Physics Today 55:12-13 (2002).
2. Zhao, F., Lai, M.-C., Harrington, D.L., Progress in Energy and Combustion Science 25:437-562 (1999).

3. Xu, M., Porter, D.L., Daniels, C.F., Panagos, G., Winkelman, J.R., Munir, K., SAE 2002-01-2746.

4. Hung, D.L.S., Mara, J.P., Winkelman, J.R., ILASS Americas, 17th Annual Conference on Liquid Atomization and Spray Systems, Arlington, VA, May 2004.

5. Gardner, R. C., Horn, W. F., Rhoades, M. K., Wells, M. D., and Yockey, S. J., "Silicon Micromachined Compound Nozzle", US Patent 4,828,184.

6. Wells, M. D., Barua, D., Richardson, W. P., Evers, L. W., "Method and Structure for Optimizing Atomization Quality of a Low Pressure Fuel Injector," US Patent 5,449,114.

7. Adrian, R.J., Ann. Rev. Fluid Mechanics 23:261-304 (1991).

8. Coil, M.A., and Farrell, P.V., SAE 950458.

9. Sick, V., and Stojkovic, B., Applied Optics 40:24352442 (2001).

10. Powell, C.F., Yue, Y., Poola, R., Wang, J., J. Synchrotron Rad. 7:356-360 (2000).

11. MacPhee, L.E., Tate, M.W., Powell, C.F., Yue, Y., Renzi, M.J., Ercan, A., Narayanan, S., Fontes, E., Walther, J., Schaller, J., Gruner, S.M., Wang, J., Science. 295:1621-1622 (2002).

12. Cai, W., Powell, C.F., Yue, Y., Narayanan, S., Wang, J, Tate, M.W., Renzi, M.J., Ercan, A., Fontes, E., Gruner, S., Applied Physics Letters 83:16711673 (2003).

13. Cheong, S.-K., Liu, J., Shu, D., Wang, J., Powell, C.F., SAE 2004-01-2026.

14. Liu, X., Liu, J., Li, X., Cheong, S.-K., Shu, D., Wang, J., Tate, M.W., Ercan, A., Schuette, D.R., Renzi, M.J., Woll, A., Gruner, S.M., 49th Annual Meeting of SPIE, (invited) Denver, CO, August 2004, 5535:2128.

15. Barna, S.L., Shepherd, J.A., Tate, M.W., Wixted, R.L., Eikenberry, E.F., Gruner, S.M., IEEE Trans. Nucl. Sci. 44:950-956 (1997).

16. Rossi, G., Renzi, M., Eikenberry, E.F., Tate, M.W., Bilderback, D., Fontes, E., Wixted, R., Barna, S.L., Gruner, S.M., J. Synchrotron Rad. 6:1096-1105 (1999).

17. Kak, A.C., and Slaney, M., Principles of Computerized Tomographic Imaging, IEEE Press, New York, 1999.

\section{CONTACT}

The corresponding author, Jin Wang, can be reached at wangj@aps.anl.gov 